\title{
Le théâtre de Juan Mayorga
}

Cartographie de la faillite humaine

\section{Gabriela Cordone}

\section{OpenEdition}

\section{Journals}

Édition électronique

URL : https://journals.openedition.org/cher/1553

DOI : $10.4000 /$ cher.1553

ISSN : 2803-5992

Éditeur

Presses universitaires de Strasbourg

\section{Édition imprimée}

Date de publication : 1 décembre 2017

Pagination : 65-77

ISBN : 978-2-86820-962-7

ISSN : 1968-035X

Référence électronique

Gabriela Cordone, "Le théâtre de Juan Mayorga », reCHERches [En ligne], 19 | 2017, mis en ligne le 01 décembre 2021, consulté le 08 février 2022. URL : http://journals.openedition.org/cher/1553; DOI : https://doi.org/10.4000/cher.1553

\section{(c) $)(1)(2)$}

Ce(tte) œuvre est mise à disposition selon les termes de la Licence Creative Commons Attribution Pas d'Utilisation Commerciale - Partage dans les Mêmes Conditions 4.0 International. 


\title{
Le théâtre de Juan Mayorga Cartographie de la faillite humaine
}

GABRIELA CORDONE ${ }^{1}$

La véritable crise, c'est l'oubli parfait, la nuit sans rêves Imre Kertész, «Ombre profonde», (2009)

\begin{abstract}
$\mathrm{D}$ ans L'Holocauste comme culture, l'écrivain hongrois Imre Kertész signale que «[1]es questions sont importantes si elles sont vitales» (2009: 90). J'aimerais placer les lignes qui vont suivre sous l'égide de cette maxime: un sujet est important s'il est vital, c'est-à-dire s'il est question de vie ou de mort, et la violence, sans doute, fait partie de ces questionnements fondamentaux. Car c'est la manifestation de la violence qui met en évidence, avec une fréquence soutenue, le fragile statut de notre culture. C'est ce qui transparait dans les œuvres majeures de Juan Mayorga - Himmelweg, Cartas de amor a Stalin, Animales nocturnos, El cartógrafo, La paz perpetua ou La tortuga de Darwin. De ces pièces, se dégage une profonde réflexion sur la violence, mais aussi sur la culture et la barbarie, le crime et la philosophie, la morale et la politique.

La nature du sujet que l'on convoque, tout autant que la complexité des textes impliqués, m’obligent à laisser de côté une exposition linéaire. En effet, la Shoah ou le totalitarisme constituent des nœuds de pensées impossibles à réduire à un aspect ou à une circonstance, à un auteur ou à une époque. Mon propos, dans un premier temps, est d'esquisser quelques pistes de réflexion autour de Himmelweg, pièce qui sera mise en relation avec les écrits du romancier hongrois Imre Kertész, survivant - le mot n'est pas exact - d'Auschwitz. Dans un deuxième temps, nous évoquerons les mécanismes totalitaires dans Cartas de amor a Stalin.
\end{abstract}

1 Maître d'enseignement et de recherche de la section d'espagnol à l'Université de Lausanne - Suisse. 
L'association de ces deux auteurs n'est ni fortuite, ni capricieuse: chez l'un comme chez l'autre, on trouve une tentative d'énoncer un discours qui n'aurait jamais dû exister. D'autres références incontournables, comme Primo Levi, ainsi que la pensée osée et lucide de Hannah Arendt, seront le fil d'Ariane dans le labyrinthe d'une lecture vitale et nécessaire. Cette approche extradramaturgique de l'œuvre de Juan Mayorga peut s'avérer - ce que j'espère productive lorsqu'on aborde les particularités de la création littéraire autour de l'expérience définitive et irréversible de l'anéantissement de l'essence humaine que constituent Auschwitz et le totalitarisme.

Dans ce parcours forcément éclectique, nous tâcherons de ne pas perdre de vue la réalité de la scène théâtrale et la particularité de la parole dramatique, objective et subjective à la fois, car elle correspond à la situation paradoxale de l'auteur-témoin - c'est-à-dire à l'objectivation difficile, à travers l'écriture, de l'expérience traumatique.

La critique et les études sur la création après Auschwitz sont nombreuses, et il ne s'agit pas, ici, de faire état des diverses postures engendrées, depuis Adorno, par l'événement éthique le plus radical de l'histoire de l'humanité2.

Interrogé sur la présence de la Shoah dans la littérature hongroise, Imre Kertész place l'imagination au centre du questionnement:

[D]ans quelle mesure l'imagination peut apprivoiser le fait de l'Holocauste, dans quelle mesure elle est capable de l'accepter et, à travers cette acceptation, dans quelle mesure l'Holocauste est devenu une part de notre vie éthique. Car c'est de cela qu'il s'agit et, si nous parlons de la littérature et de l'Holocauste, c'est de cela qu'il faut parler. (2009: 53)

Et il ajoute un peu plus loin: «Nous connaissons tous la célèbre phrase d'Adorno: on ne peut plus écrire de poésie après Auschwitz», mais l'écrivain apporte une nuance significative à ce postulat, en proposant "qu'après Auschwitz, on ne

2 Parmi les études sur le sujet, on peut se référer à Dienberg Thomas, 1997, Ihre Tränen sind wie Gebete: das Gebet nach Auschwitz in Theologie und Literatur, Würzburg, Echter; Dunker Axel, 2003, Die anwesende Abwesenheit: Literatur im Schatten von Auschwitz, München, W. Fink; Falconer Rachel, 2007, Hell in contemporary literature: Western descent narratives since 1945, Edinburgh, Edinburgh University Press; Garscha Karsten et al., 2006, Écrire après Auschwitz: mémoires croisées France-Allemagne, Lyon, Presses universitaires de Lyon; Grierson Karla, 2003, Discours d'Auschwitz: littérarité, représentation, symbolisation, Paris, H. Champion; Genève Slatkine et Hirsch David H., 1991, The deconstruction of literature: criticism after Auschwitz, Hanover New England, London, Brown univ. Press; Kiedaisch Petra (hrsg.), 1998, Lyrik nach Auschwitz?: Adorno und die Dichter, Stuttgart, Reclam, 1998; Köppen Manuel (hrsg.), 1993, Kunst und Literatur nach Auschwitz, Berlin, E. Schmidt; Mertens Pierre, 2003, Écrire après Auschwitz?: Semprun, Levi, Cayrol, Kertész, Tournai, La Renaissance du Livre; Mosler Peter (hrsg.), 1989, Schreiben nach Auschwitz, Köln, Bund-Verlag; Réra Nathan, 2009, De Paris à Drancy ou Les possibilités de l'art après Auschwitz, Pertuis, Rouge profond; Schulte Nordholt, Annelise (éd.), 2008, Témoignages de l'après-Auschwitz dans la littérature juivefrançaise d'aujourd'hui: enfants de survivants et survivants-enfants, Amsterdam, Rodopi; Trigano Shmuel (dir.), 1989, Penser Auschwitz, Paris, Cerf. 
peut plus écrire de poésie que sur Auschwitz» (2009: 54), plaçant l'événement au centre de la mémoire.

En ce sens, l'œuvre de Juan Mayorga est un acte permanent de mémoire et une lutte contre l'oubli, car «después de Auschwitz [el mundo] ha seguido como si nada hubiera ocurrido, cabe sospechar que nuestro mundo es contemporáneo de quienes vivieron los momentos previos a la catástrofe» (Mate y Mayorga 2000: 46). Ceci explique l'urgence, chez le dramaturge madrilène, d'une écriture centrée sur Auschwitz et le totalitarisme, comme une manière d'assumer ainsi le poids des événements et l'effort de compréhension, dont Hannah Arendt dira:

[comprendre] veut plutôt dire examiner et porter en toute conscience le fardeau que les événements nous ont imposé, sans nier leur existence ni accepter passivement leur poids comme si ce qui est arrivé en fait devait fatalement arriver. Comprendre, en un mot, consiste à regarder la réalité en face avec attention, sans idée préconçue, et a lui résister au besoin, quelle que soit ou qu'ait pu être cette réalité. (1984: 16-17)

Tout comme Kertész, Améry ou Levi, Juan Mayorga s'est aussi imposé la tâche de penser la violence politique, et il me semble qu'il s'agit du dénominateur commun de ses pièces les plus significatives. Mais comment dire la violence en scène, avec quels mots, à travers quels personnages? En d'autres termes, existe-il une «communicabilité esthétique de la violence»? (Kertész 2001: 45).

Il est évident que les positions des auteurs que nous venons d'évoquer face à la création littéraire sont très différentes: Kertész, Améry ou Levi écrivent comme témoins, à partir d'une expérience personnelle. Proposer ici une approche associant les survivants et un dramaturge espagnol du $\mathrm{XXI}^{\mathrm{e}}$ siècle peut sembler anachronique. Pourtant, nous aimerions, par cette démarche nonconventionnelle, suivre le chemin tracé par Aurélia Kalisky dans son étude sur Kertész et Améry (Kalisky 2006): en proposant un dialogue entre Mayorga et les témoins directs, nous visons aussi, comme l'exprime la chercheuse, l'idée de transmission, "d'un passage de témoignage ${ }^{3}$ qui devienne une valeur, et la valeur elle-même "une force spirituelle créatrice de loi" au sens moral, éthique et politique» (2006:57).

Recevoir, accueillir et transmettre le témoignage - telle est la tâche que s'est imposée Juan Mayorga - n'est pas sans difficultés. Le témoignage personnel, traditionnellement, n'a pas le même poids qu'un document historique. En ce sens, Imre Kertész se demande si la culture est capable de reconnaître un type de savoir autre que celui que l'on appelle «objectif», car la pensée occidentale n'a jamais accordé de valeur quelconque à l'expérience subjective. Ainsi, dans "L'intellectuel superflu», Kertész attribue les crimes historiques du $\mathrm{xx}^{\mathrm{e}}$ siècle à l'abstraction menée à l'extrême: "Croyez-moi, les crimes historiques de ce siècle ont été causés en grande partie par cette abstraction poussée à l'extrême, cette fureur de réflexion presque pathologique et l'absence totale d'imagination qui s'ensuit»(2009: 94). En effet, comme le signale Kalisky à propos de

3 Presque comme si l'on parlait de passage de témoin (Kalisky 2006: 57, note 8). 
Kertész, l'une des origines du caractère destructeur des idéologies en acte réside "dans cette négation de l'expérience de l'individu et cette toute-puissance de l'objectivation» (2006: 58). Ces propos doivent être mis en rapport avec une figure moins connue de la pensée allemande du début du $\mathrm{xx}^{\mathrm{e}}$ siècle, celle de Franz Rosenszweig, dont Mayorga fait une lecture lucide et attentive:

Rosenszweig es el pionero, el primero en denunciar que la metafísica occidental era una ontología de la guerra. [...] Su notable contribución comienza a) cuando califica de «idealismo» no al Idealismo Alemán, sino a toda la filosofía europea, la que va «desde los jónicos a Jena», es decir, desde los presocráticos hasta Hegel [...] Y, b) cuando coloca a la experiencia pertinaz de la angustia ante la muerte como el argumento desencadenante de toda su descalificación del idealismo. (Mate et Mayorga 2000: 46-47)

La négation de l'expérience individuelle évoquée par Kertész se retrouve, dans la réflexion de Mayorga et Mate, au sujet de Rosenzweig dans L'Étoile de la rédemption. En effet, Rosenzweig signale, à propos de l'idéalisme, le problème qui consiste à vouloir réconcilier deux points fondamentaux comme la raison et la mort. Pour l'idéalisme, le non-sens de la mort individuelle trouve son sens dans le Tout auquel l'être appartient. Malgré ce raisonnement, conclut Rosenzweig, l'angoisse de la mort est une expérience toujours nouvelle, et le néant de la mort est toujours quelque chose. Par ailleurs: "[u]na filosofía que coquetea así con la muerte, es potencialmente una justificación del crimen. Si, filosóficamente, la muerte individual carece de sentido, ¿qué impide el crimen político?» (Mate y Mayorga 2000: 49). Rejoignant Kertész en ce qui concerne la place de l'expérience, Mayorga voit dans l'idéalisme occidental, à travers Rosenzweig, la négation de la réalité et, surtout, de la singularité.

Si la reconnaissance de l'expérience individuelle semble, de nos jours, acquise ${ }^{4}$, des auteurs comme Kertész, survivant d'Auschwitz, ont dû se confronter durant des décennies à la négation de la légitimité de leur subjectivité, qui en aucun cas ne pouvait atteindre la portée d'une donnée dite objective. En ce sens, Aurélia Kalisky souligne le désespoir et la frustration des rescapés dont les témoignages personnels, dans les années 1970, peinaient à être reconnus comme ayant une autorité spécifique (2000: 76). Car la culture, signale Imre Kertész, est une "conscience qui objective», qui ne peut pas intégrer le savoir subjectif des survivants, et Kalisky d'évoquer, dans une étude postérieure, la manière dont le témoin peut s'approprier ce «je» que la culture cherche à lui dérober.

Selon leurs témoignages, pour Imre Kertész comme pour Jean Améry et Primo Levi, la vie après Auschwitz est invivable. L'écriture, dans ces trois cas - mais aussi chez Jorge Semprún ou Robert Antelme - se trouve intimement liée à la question de la survie. L'auteur hongrois essayera, à travers l'écriture, de s'interroger sur la communicabilité éthique de la violence: il s'agit, comme l'explique Kalisky (2000: 78), de reconquérir et de s'approprier le droit à

4 Voir Wieviorka (1998). 
l'objectivation ici même où l'histoire - Auschwitz - s'est emparée de son individualité.

Dans l'écriture produite par un témoin se pose, néanmoins, un problème majeur: comment rapprocher l'abyme qui sépare le point de vue d'un narrateur et l'expérience subjective du vécu? De quelle manière incorporer une histoire personnelle sans trahir, par l'objectivation, l'expérience du «je»? Kertész pose la question du témoignage, de la transmission et de sa forme littéraire dans $L e$ refus:

Moi aussi je voulais faire passer un message, sinon je n'aurais pas écrit de roman. Communiquer, à ma façon, selon mes idées, communiquer le matériau qu'il m'est possible de transmettre, mon expérience, moi-même, car tendu et alourdi par son poids comme une mamelle gonflée qui attend la traite libératrice, j'aspirais à communiquer... Sauf que, peut-être naturellement, je n'avais pas pensé à une chose: on ne peut jamais se communiquer à soi-même. Moi, je n'avais pas été emmené à Auschwitz par le train du roman, mais par le vrai. (2001: 74)

Différés dans l'espace et dans le temps, les textes du dramaturge madrilène Juan Mayorga posent des questions intimement liées aux précédentes: comment représenter (dans le sens de présenter encore une fois ou de rendre présent), plus de soixante ans après les évènements, la Shoah? Quelles instances d'énonciation doivent être sollicitées et quel point de vue devrait être adopté pour communiquer, depuis la scène, l'événement qui marque la fin de l'humanité? Comment, finalement, envisager l'énonciation esthétique de la violence?

Les problèmes d'énonciation, comme le signale Ubersfeld, ne sont pas simples et l'écriture théâtrale ne simplifie pas les choses: l'énoncé, au théâtre, dépend des conditions d'énonciation qui lui donnent un sens particulier (1996: 186). Le discours théâtral lui-même pose, de son côté, des interrogations liées à un-e auteur-e hors texte et au statut mouvant du personnage: équivoque et contradiction semblent constitutifs d'un discours où le sujet - et ceci est fondamental pour notre propos - cesse d'être source de sens. Sans résoudre le problème insurmontable posé par Kertész quelques lignes plus haut, le discours théâtral peut assumer le caractère non-individuel d'un énoncé et revêtir ainsi un intérêt particulier pour faire circuler l'expérience du personnage dans une scène objectivante.

Mais revenons au sujet qui nous occupe: concrètement, les pièces qui mettent en scène la violence d'État - Auschwitz, le stalinisme - ont recours à une instance énonciatrice variable qui va de l'expérience du sujet-personnage à l'objectivation d'un personnage-narrateur. Himmelweg oscille entre le témoignage contradictoire mais raisonné de l'envoyé de la Croix-Rouge et les interventions abruptes de Gershom; Cartas de amor a Stalin est rythmé par un discours épistolaire certes objectivant et prolixe, mais qui scande cependant l'anéantissement de Bulgákov, de la même manière que les minutieuses cartes géographiques assument la destruction physique et mentale dans El cartógrafo.

Ces deux constantes - expérience et pensée - échafaudent le théâtre de Juan Mayorga et lui font revêtir un caractère profondément politique, et c'est sous 
cet aspect-là que l'on peut rendre compte des mécanismes de la violence, de son agencement sur la scène et de ses conséquences éthiques.

Pour pouvoir aborder, ne serait-ce que partiellement, le mécanisme de la violence dans le théâtre de Mayorga, les réflexions de Hannah Arendt à ce sujet nous ont été d'une grande aide. Dans Les origines du totalitarisme, Arendt analyse ce qu'elle appelle «les trois piliers de l'enfer»: l'antisémitisme, l'impérialisme et le racisme, ce que nous trouvons parfaitement concrétisé dans des pièces comme Himmelweg, El cartógrafo, Cartas de amor a Stalin et Animales nocturnos.

Comme nous l'avons mentionné plus haut, Juan Mayorga s'est penché sur l'étude de l'idéalisme de la philosophie européenne et souligne concernant ce courant la négation de la pluralité existante, sa «querencia insuperable a reducir lo múltiple al uno" (Mate y Mayorga 2000: 47) et le situe à la base de la structure démentielle qui a érigé les camps de concentration et d'extermination allemands. La pensée philosophique, à partir de cet instant, cesse d'offrir un cadre pertinent pour appréhender la réalité humaine. Il s'agit donc, dans les œuvres évoquées, non d'une réflexion sur un sujet douloureux, mais de l'expérience d'une rupture totale. Comme l'exprime Arendt, "[1]a terrible originalité du totalitarisme ne tient pas au fait qu'une "idée" nouvelle soit venue au monde, mais à ce que les actions mêmes qu'elle a inspirées constituent une rupture par rapport à toutes nos traditions: ces actions ont manifestement pulvérisé nos catégories politiques, ainsi que nos critères de jugement moral» (1990: 42).

De quelle manière, avec quels mots et avec quels gestes Juan Mayorga dit la violence? Aucune des œuvres mentionnées - Himmelweg, El cartógrafo, Cartas de amor a Stalin, Animales nocturnos - ne contient des scènes de violence. Nous sommes loin du spectacle de la bestialité - comme dans le sanguinaire Titus Andronicus shakespearien - mais nous sommes plutôt confronté-e-s à des espaces feutrés de la vie quotidienne: le bureau d'un fonctionnaire, une bibliothèque, la salle de travail d'un écrivain, un bistrot de quartier... Même les mots sont intentionnés et doux («Puis-je m'asseoir à votre table?» «Désirezvous encore un café?»). Pourtant, nous savons que les espaces domestiques sont les plus perméables à la violence: Mayorga choisit cette quiétude apparente pour déplier, avec des mots et des silences seulement, l'abyme de l'horreur. Sans coups, ni cris, ni pleurs - gestes associés à la manifestation de la violence -, une pièce comme Himmelweg retrace le principe même de l'abominable processus de deshumanisation qui a modifié, à tout jamais, l'essence de l'humain.

Dans cette pièce, l'énoncé est construit à partir d'une contradiction: les paroles d'un témoin externe, censé voir l'horreur mais qui n'a rien vu. Il s'agit d'une sorte d'anti-témoin, dont le discours est fait de lacunes, d'approximations et de maladresses. Ce qu'il ne dit pas, qu'il dit à moitié ou qu'il tait - les bruits et le silence - évoquent, néanmoins, des évènements qui par leur violence inouïe ont transformé toutes nos catégories de pensée.

Les nazis ont été les maîtres de la falsification et de la farce. Primo Levi fait allusion, dans le premier de ses récits, aux pratiques et noms instaurés par les 
Allemands dans les camps d'extermination. Ainsi, l'un des euphémismes les plus significatifs et atroces fut le «chemin du ciel» - Himmelweg - pour se référer aux cheminées des fours crématoires (Agamben 2003: 87).

Ils furent aussi maîtres de la mise en scène, comme on peut le déduire de l'entretien de Maurice Rossel - envoyé de la Croix-Rouge auprès du camp de Theresienstadt - accordé au cinéaste Claude Lanzman en 1979 et dont le récit a servi de base à la pièce théâtrale qui nous occupe. En effet, l'action - tant dans les faits historiques que dans son récit - se trouve prisonnière de la fiction dès le début: la réalité historique fut une farce macabre. La visite de l'observateur de la Croix-Rouge au camp de Theresienstadt avait été préparée des mois à l'avance, avec une scénographie spécifique pour recréer un village juif modèle, des répétitions des interné-e-s dans leur rôle et des répétitions générales. Historiquement, rien n'était vrai. Sur la scène, rien n'est fiction: cette pièce, fortement associée à l'entretien filmé par Lanzman, appelle à une réflexion de fond sur la violence sourde et muette qui bouge, sous nos yeux, les ficelles des marionnettes vouées à la mort.

Arrêtons-nous, pour ce faire, sur quelques passages. Lus sous le regard de Hannah Arendt, des phrases en apparence sans importance prennent lugubrement toute leur signification: «[El encargado de la Cruz Roja]: De vez en cuando, alguien entra con un expediente. Todo es como en una oficina. Ellos parecen estar haciendo algo útil» (Mayorga 2011: 132).

La grande nouveauté du phénomène totalitaire est le crime administratif, planifié, rationnalisé et exécuté par des fonctionnaires consciencieux, employés auprès d'une administration bureaucratique qui a pour but l'articulation optimale de la production en masse de la mort avec une réduction significative des dépenses. Arendt avait défini, dans son étude Les origines du totalitarisme, «le mal absolu» comme la fabrication industrielle de cadavres. Plus tard, dans Eichmann à Jérusalem, la philosophe se centre sur la banalité du mal, et décrit Eichmann non pas comme un monstre sanguinaire et démoniaque, mais plutôt comme un criminel de bureau ${ }^{5}$. Mayorga reprend ce profil dont Arendt rend compte de la simplicité de fonctionnement, et l'agrémente des traits qui ont à voir davantage avec la pensée de Walter Benjamin. Le Commandant de

5 Cependant, comme Erwan Burel me l'a très pertinemment signalé, des travaux récents ont démontré la fragilité de la thèse arendtienne: par exemple, la biographie d'Adolf Eichmann réalisée par David Cesarini (Adolf Eichmann. Comment un homme ordinaire devient un meurtrier de masse, Talandier, 2004), où il est fait mention de nombreux épisodes où l'homme nazi apparaît sous un tout autre jour que lors de son procès. Cesarini se penche notamment sur plusieurs annotations marginales des livres qu'Eichmann lisait durant son exil argentin, ainsi que sur divers entretiens qu'il avait accordés au vétéran SS Willem Sassen. La conclusion est sans appel: Eichmann apparaît comme un véritable assassin de masse convaincu. Le procès de Jérusalem de 1961 a, en réalité, engendré une catégorie - celle du criminel de bureau, dissocié des conséquences de ses actes et de ses décisions, inconscient du crime dont il participe pourtant pleinement - dans laquelle Adolf Eichmann s'est inscrit avec une grande hypocrisie, pensant se sauver de la mort. 
Himmelweg est un homme de culture: «[El encargado de la Cruz Roja]: Señalaba su biblioteca: "Calderón, Corneille, Shakespeare... Esto es Europa para mí.” » (Mayorga 2011: 133).

Les classiques de l'art dramatique se sont donné rendez-vous dans les étagères d'une dépendance administrative d'un camp de concentration... Mise à part l'allusion, à travers ces auteurs, à la magistrale fiction théâtrale à laquelle l'observateur de la Croix-Rouge assiste, apparemment à son insu, la démonstration du patrimoine culturel dont fait preuve le SS renvoie sans doute à Walter Benjamin et aux thèses Sur le concept d'histoire: "[c]ar il n'est pas un témoignage de culture qui ne soit pas en même temps un témoignage de barbarie»(Benjamin, thèse VII). Les deux personnages de ce jeu d'échecs, le Commandant et le Délégué de la Croix-Rouge, montrent à quel point la violence totalitaire a profondément affecté la capacité de jugement ou, comme le suggère Arendt, a révélé son état d'effondrement non seulement concernant les élites allemandes mais aussi européennes. Cette violence pose également la question, aujourd'hui, du point de vue éthique, de notre responsabilité en tant qu'individus et en tant que polis dans les situations de crise. Ainsi, la violence implicite qui se dégage du Commandant trouve son pôle négatif - grâce auquel le courant passe, si nous filons la métaphore - dans le Délégué de la Croix-Rouge: Himmelweg, à mon avis, montre aussi du doigt l'ignominie de la communauté internationale qui, elle non plus, n'a pas voulu voir et a participé, en détournant son regard, à la mise en place de la solution finale. Tout comme le Délégué de la Croix-Rouge, qui a peut-être été victime, dans son aveuglement, de son propre antisémitisme (Fontaines 1997).

Himmelweg est une représentation d'une représentation. L'automatisme des mouvements et des déplacements des «acteurs", tant dans l'histoire que dans la fiction, trouve son pendant dans le discours de Gottfried. La pièce dévoile déjà le fonctionnement mécanique de la farce qui est en train de se jouer, présidée par l'horloge de la gare «construido hacia el año mil quinientos dos por el maestro Peter Henlein, de Nuremberg, el famoso fabricante de juguetes automáticos » (Mayorga 2011: 135). Jouets automatisés, mécanisme horloger... tout met en évidence l'artificialité de la situation mais aussi la précision démentielle avec laquelle l'industrie de l'extermination est mise en marche.

Maurice Rossel, lors de son entretien avec Claude Lanzman, qualifie les interné-e-s de "marionnettes» ou "automates». Au-delà de la correspondance des termes employés par Rossel, dans Himmelweg ces mots retentissent autrement, car ils mènent à l'idée de la destruction humaine. Jean-Claude Poizat, à propos de la survie dans les camps, signale que la vie

se voit ramenée en effet à de simples fonctions mécaniques. Le combat pour la survie est dénué de sens et inutile, il est réduit à une caricature où les détenus, instrumentalisés, sont comparables à des marionnettes. Ils sont maintenus dans une sorte d'entre-deux, dans un temps d'avant la naissance et d'après la mort, dans une «vie après la mort» qui fait d'eux des cadavres vivants. (Poizat 2013: 76) 
La présence de l'horloge mécanique arrêtée macabrement à six heures du matin, au moment de l'arrivée des nouveaux et des nouvelles interné-e-s qui prendront le chemin $\mathrm{du}$ ciel, ainsi que les scènes et les mouvements répétés pour tenter d'assurer une éventuelle survie sont autant de ruptures de l'humain, fruit non pas d'une folie meurtrière mais d'un plan articulé intellectuellement et mis en place de manière méthodique et raisonnée. Les camps de concentration et d'extermination des régimes totalitaires servent, pour Arendt, "de laboratoires où la croyance fondamentale du totalitarisme - tout est possible - se trouve vérifiée» (1990: 173). L'affirmation est reprise par Mayorga dans le monologue du Commandant adressé aux spectateurs - qui prennent la place, parfois, des délégué-e-s de la Croix-Rouge et, me semble-t-il, des anciens et des anciennes déporté-e-s:

El objetivo inmediato es reagrupar aquí a todos los hebreos de Europa. Pero nuestro objetivo final es mucho más elevado. Nuestro objetivo final es demostrar que todo es posible. Todo es posible. Todo lo que podamos soñar, podemos hacerlo realidad. Aquí, en este mundo. Incluso lo que nunca nos hemos atrevido a imaginar. Eso es, señoras y señores, lo que les aguarda en el bosque: aquello que se puede ver, pero que no se puede imaginar. (Mayorga 2011:151)

De l'inimaginable nous parle également Primo Levi dans sa Préface de Les naufragés et les rescapés:

Les premières informations sur les camps d'extermination nazis ont commencé à se répandre en 1942, année cruciale. Elles étaient vagues, elles concordaient toutefois pour ébaucher l'image d'un massacre de dimensions tellement vastes, d'une cruauté poussée si loin, aux motivations tellement complexes, que le public avait tendance à les repousser en raison même de leur énormité. (1986:11)

Le Commandant mayorguien réunit en lui les multiples facettes des individus qui ont rendu possible le cauchemar nazi et revêt, dans la pièce, les caractéristiques d'une sorte de présentateur de show de pacotille. Une des facettes est celle de l'intellectuel de droite, porteur d'un discours creux et dangereux, grandiloquent et destructif. L'avènement d'un monde nouveau, possible grâce au courage dont devront faire preuve quelques individus, est au centre d'une argumentation soutenue par l'Idéalisme tant critiqué par Kertész et notre dramaturge:

Es mejor sufrir mil años que regresar un instante al mundo viejo. Pasar de un mundo a otro exigirá de todos un enorme coraje. Coraje para hacer lo necesario. Necesariamente, muchos hombres caerán en el camino, ellos son parte del camino. Ellos son el camino. (Mayorga 2011: 150)

Remarquons, en passant, la concaténation comme paradigme des répétitions qui caractérisent les discours politiques vides de sens. Encore une fois, Walter Benjamin et les thèses de philosophie de l'histoire se profilent derrière ces mots. L'idée de progrès, tant critiquée par Benjamin, peut se lire en filigrane. Sa pensée se distingue en ce qu'elle ne considère pas que l'humanité progresse. La douleur et les souffrances humaines, soutient Benjamin, ne peuvent jamais être justifiées en vue d'asseoir le progrès, comme nous avons pu le montrer à propos 
d'une autre pièce du dramaturge madrilène, La tortuga de Darwin (Cordone 2011: 147).

D'autre part, la nature histrionique du Commandant renvoie aussi au profil du fonctionnaire zélé, qui veut répondre correctement à la «sollicitation de Berlin» (Mayorga 2011: 154), le bourgeois déclassé, cynique et arrogant à la base de la main-d'œuvre soumise et efficace de la machine totalitaire, dont le personnage Hombre Bajo dans Animales nocturnos est l'exemple parfait. Hannah Arendt analyse les traits de cet «homme superflu» dans ces termes:

Pour les impitoyables machines de domination et d'extermination, la masse coordonnée de philistins était un matériau bien meilleur et capable de crimes bien plus grands que les soi-disant criminels professionnels, pourvu que ces crimes fussent soigneusement organisés et eussent l'apparence de travaux de routine. (Arendt 1972: 65)

Aux traits de l'intellectuel de foire, Mayorga ajoute ceux du fonctionnaire dévoué - transposé en directeur de scène névrosé - obnubilé dans son verbiage vain et futile. Et le Commandant d'ajouter, au comble de l'ironie: "Honrados padres de familia se convierten en bestias en cuanto se ven con este uniforme» (Mayorga 2011: 154). Le discours idéologique fonctionne comme un écran entre la conscience et la réalité et traduit l'incapacité de penser par ses propres moyens et de considérer le point de vue de l'autre. Absorbé par sa mission, le Commandant est le paradigme de l'abrutissement de l'appareil nazi au service de l'anéantissement de l'être humain'.

Un passage de Kertész synthétise de manière magistrale une pensée que nous pouvons appliquer à la composition intrinsèque de ce personnage: "Oui, poursuivais-je ma réflexion, c'est peut-être là que se cache le diable: non dans le fait que l'homme tue, mais dans celui que les vertus indispensables au crime deviennent pour lui l'ordre du monde» (Kertész 2001 : 51).

Cartas de amor a Stalin complète le cadre de cette approche du traitement de la violence chez Juan Mayorga. Il s'agit de l'anéantissement de la liberté individuelle et créatrice. Nous trouvons, dans les écrits d'Hannah Arendt, la description et l'analyse d'une expérience, fruit de l'émergence de la société de masse, la désolation, qu'elle définit comme la perte du monde ou la destruction de l'appartenance au monde. Paradoxalement, signale Poizat, l'expérience de la désolation est une non-expérience, «dans la mesure où elle résulte de la destruction du rapport à la communauté humaine» (2013: 69). Afin de saisir cette condition particulière, le chercheur propose de les comparer à deux

6 Lorsque Hannah Arendt parle d' «homme superflu», elle fait référence aussi bien au serviteur du régime totalitaire qu'à "l'homme de masse», victime du régime totalitaire, dont les dimensions juridique, morale et individuelle ont été anéanties, rendant sa personne superflue et «permettant» son extermination. Poizat précise aussi que cela «[...] explique notamment l'un des traits les plus caractéristiques des régimes totalitaires: le fait que ceux qui en ont été les serviteurs zélés, notamment les fonctionnaires déshumanisés des camps de concentration, se considéraient euxmêmes, à l'instar de leur victimes, comme des "hommes superflus" " (2003: 54). 
autres expériences, celle de la solitude et celle de l'isolement. Pour la lecture de Cartas de amor a Stalin, ces trois catégories vont se révéler essentielles pour comprendre le dessein graduel de la démolition du protagoniste, l'écrivain russe Mijaíl Bulgákov.

La solitude est une séparation provisoire de l'entourage, qui permet de prendre de la distance et de se ressourcer pour mieux revenir vers la communauté. C'est l'expérience de l'écrivain, seul dans sa bibliothèque ou dans une salle, qui, dans sa retraite, crée pour un public de lecteurs ou spectateurs potentiels: «Tú eres el escritor. Conoces el efecto de las palabras sobre la gente» (Mayorga 2014: 223). Arendt considère cette expérience de la solitude de manière positive: "[1]a solitude institue un rapport de soi à soi-même qui est une sorte de dédoublement du moi [...] il s'agit [...] de se retrouver avec soi-même pour mieux rejoindre [la communauté]» (Poizat 2013: 70).

Dès les scènes initiales de Cartas de amor a Stalin, ce premier état de grâce est perdu et le conflit se noue à partir de l'effort pour réintégrer la communauté, proche ou lointaine:

Bulgákov (Leyendo.) - «Estimado camarada: Mi obra La huida, cuyo estreno estaba previsto para el próximo septiembre, ha sido prohibida durante los ensayos. Las representaciones de La Isla Púrpura han sido prohibidas. Los días de los Turbin, después de trescientas representaciones, ha sido prohibida. El apartamento de Zoika, después de doscientas representaciones, ha sido prohibida. Así pues, mis cuatro obras teatrales se encuentran prohibidas. La edición de mis relatos ha sido prohibida, igual que han sido prohibidos mis ensayos. La lectura pública de Las aventuras de Chichikov ha sido prohibida. La publicación de mi novela La guardia blanca en la revista Rossia ha sido prohibida. No tengo ánimos para vivir en un país en el que no puedo ni representar ni publicar mis obras. Me dirijo a usted para pedirle que se me devuelva mi libertad como escritor (Pausa.) o se me expulse de la Unión Soviética junto con mi esposa». (Mayorga 2014:221-222)

C'est l'expérience de l'isolement, telle qu'elle est conçue par Arendt, qui marque le début de cette pièce. "L'isolement est cette impasse où sont conduits les hommes lorsque la sphère politique de leurs vies, où ils agissent ensemble dans la poursuite d'une entreprise commune, est détruite» (Arendt 1972: 228). Le personnage de Bulgákov se trouve dans cette situation de mise à l'écart. Il possède encore, néanmoins, la faculté d'agir. Il écrit, il s'exprime, il crée. Cette activité - l'écriture - lui est nécessaire. Les lettres adressées au tyran sont une façon d'être en lien avec l'autre. Certes, il s'agit d'un rapport individuel pour solliciter la réintégration dans les rapports politiques. Assigné à l'isolement et exclu des rapports à la communauté, Bulgákov se réfugie dans une production vaine qui gagne, peu à peu, son esprit, et le conduit au domaine de la désolation ou, comme on disait plus haut, de la destruction de l'appartenance au monde. Jean-Claude Poizat commente cet état dans ces termes:

Ainsi, l'ultime étape de la destitution de l'humain est le résultat de l'anéantissement total des conditions de possibilité de l'être-ensemble. Elle est la conséquence de la destruction totale de la relation individuelle à l'espace commun. Bien plus, la 
désolation révèle une incapacité à entrer en relation avec soi-même par la pensée. (Poizat 2013: 72)

C'est bien par ce processus destructeur que le protagoniste de Cartas de amor a Stalin s'éloigne de plus en plus de lui-même, jusqu'à la perte totale de ses repères, du dédoublement intime et monstrueux avec son bourreau, de l'abandon du seul être humain qui faisait encore de lui un homme.

En définitive, ne cherchons pas dans le théâtre de Juan Mayorga des expressions d'indignation ou de réprobation morale devant la violence totalitaire. Nous n'allons pas trouver ce type de considération, car les valeurs humanistes ont cessé d'être adaptées pour lire et interpréter les faits montrés sur scène. En effet, la violence totalitaire inaugure un autre genre de violence, sans commune mesure, car, comme l'explique la philosophe, "en s'efforçant de prouver que tout est possible, les régimes totalitaires ont révélé sans le savoir l'existence de crimes que les hommes ne peuvent ni punir ni pardonner» (Arendt 1972: 200).

Aux penseurs et penseuses, aux artistes et aux écrivain-e-s revient la tâche de rendre encore une fois ces faits au public pour qu'ils puissent être jugés, car tout se joue dans l'espace public. Par-dessus toutes les caractéristiques humaines, la liberté politique est celle qui semble la plus essentielle à l'être humain. Une œuvre comme Animales nocturnos, qui touche la question des sans-papiers, se trouve ainsi en plein cœur de la mécanique totalitaire ${ }^{7}$.

En nous appuyant sur des textes venant d'autres horizons, la dimension politique du théâtre de Juan Mayorga surgit avec une force renouvelée.

Replacer le théâtre au centre de la polis est une nécessité pour le dramaturge madrilène. Il est évident que nous vivons une époque où les normes établies vont contre les droits politiques et la dignité humaine. Mayorga en appelle à notre capacité de jugement, car c'est notre seule forme de résistance. Pour le philosophe et le dramaturge, revenir sur Auschwitz et le totalitarisme signifie certainement s'imposer un travail d'objectivation et de compréhension qui, comme le rappelle Arendt, a pour but d'examiner et d'assumer en toute conscience le poids que les évènements ont imposé.

[L]es humanistes professionnels voudraient croire qu'Auschwitz est arrivé uniquement à ceux auxquels il est arrivé précisément à cet endroit-là, en ce temps-là, mais ceux auxquels il n'est pas arrivé précisément à cet endroit-là et en ce temps-là, c'est-à-dire à la plupart des autres gens - l'Homme! - eh bien, il ne leur est rien arrivé du tout. (Kertész 2001: 40)

Seule l'imagination littéraire peut rendre compte des faits qui sont devenus partie intégrante d'une logique antihumaine, en vertu de laquelle les êtreshumains ont été dépossédés de leurs droits, et le fait de commettre un crime à leur encontre devient la norme. En ce sens, il nous semble que le théâtre de

7 Pour Arendt, le «petit homme» représente «l'homme de masse» qui vit dans un monde de désolation. Rappelons que Mayorga nomme "el Hombre Bajo" le personnage médiocre qui exerce son pouvoir sur le sans-papiers. 
Mayorga est en profonde résonance avec la pensée de Arendt et de Kertész, car il assume les conséquences éthiques d'Auschwitz et les assume comme un vécu universel, qui dépasse le simple conflit entre Allemands et Juifs. Tout comme Roberto Benigni, Juan Mayorga «représente une nouvelle génération aux prises avec Auschwitz, qui a le courage et la force de revendiquer ce funeste héritage» (Kertész 2009 : 159).

\section{Bibliographie}

Agamben G., 2003, Ce qui reste d'Auschwitz, Paris, Payot.

Arendt H., 1972, Le Système totalitaire, Paris, Seuil.

Arendt H., 1984, Sur l'antisémitisme, Paris, Seuil.

Arendt H., 1990, La Nature du totalitarisme, Paris, Payot.

Benjamin W., 1973 [1940], Tesis de filosofía de la historia, Jesús Aguirre (trad.), Madrid, Taurus.

Kalisky A., 2006, «Avis de liquidation / Inventaire avant démolition. Jean Améry en dialogue avec Imre Kertész », in : Jürgen Doll (éd.), Jean Améry (1912-1978). De l'expérience des camps à l'écriture engagée, Paris, L'Harmattan, p. 55-83.

Kertész I., 2001, Le Refus, Paris, Actes Sud.

Kertész I., 2009, L'Holocauste comme culture, Paris, Actes Sud.

Fontaines P., 1997, «Un Vivant qui passe», http://www.lesinrocks.com/cinema/ films-a-l-affiche/un-vivant-qui-passe/ [Consulté le 19/12/2016].

Lanzman C., 1979, Un Vivant qui passe, http://www.ushmm.org/online/film/ display/detail.php?file_num=5012 [Consulté le 19/12/2106]

Levi P., 1986, Les naufragés et les rescapés. Quarante ans après Auschwitz, Paris, Arcades Gallimard.

Levi P., 1987, Si c'est un homme, Paris, Julliard.

Mayorga J., 2011, Himmelweg, Manuel Aznar Soler (ed.), Ciudad Real, Naque.

Mayorga J., 2014, Cartas de amor a Stalin, in: Teatro 1989-2014, Segovia, La uÑa RoTa, p. 219-258.

Poizat J.-C., 2013, Hannah Arendt, une introduction, Paris, Pocket.

Wieviorka A., 1998, L'ère du témoin, Paris, Plon. 